\title{
Efectos de la exposición a música activante y relajonte sobre el estado emocional de una población de adolescentes
}

\author{
Veronika Diaz Abrahan* \\ Flavia Gatto** \\ Nadia Justel ***
}

Recibido en 29 de julio de 2019, aceptado en 26 de agosto de 2020

\section{Citar este artículo así:}

Diaz-Abrahan V, Gatto F, Justel N. Efectos de la exposición a música activante y relajante sobre el estado emocional de una población de adolescentes. Hacia. Promoc. Salud. 2021; 26 (1): 84-97 DOI: 10.17151/hpsal.2021.26.1.8

\begin{abstract}
Resumen
La ansiedad es definida como la anticipación a una futura amenaza, con una prevalencia que alcanza el 8\% en las personas jóvenes a nivel mundial. Objetivo. El propósito de este trabajo fue identificar los efectos de la exposición a música sobre la ansiedad en una muestra de individuos adolescentes entre 10 y 14 años de ambos sexos. Materiales y métodos. Cada uno de los participantes completó un test estandarizado para evaluar su ansiedad. De modo previo a los tratamientos, los participantes completaron las escalas de estado y rasgo del STAI, luego escucharon estímulos sonoros (música relajante, activante o ruido blanco) y finalmente completaron la escala de estado de ansiedad. Resultados. Los resultados indicaron que el estímulo musical con características activantes generó un efecto ansiogénico en los participantes. Conclusiones. Estos datos son relevantes para el campo de la musicoterapia, disciplina que utiliza la música con fines terapéuticos y que aborda los trastornos de ansiedad en diferentes poblaciones.
\end{abstract}

\section{Palabras clave}

Ansiedad; música; activante; relajante; adolescentes (fuente: DeCS, BIREME).

\footnotetext{
* Licenciada en Musicoterapia. Doctora en Neurociencias. Laboratorio Interdisciplinario de Neurociencia Cognitiva (LINC). Centro de Estudios Multidisciplinarios en Sistemas Complejos y Ciencias del Cerebro (CEMSC3), Escuela de Ciencia y Tecnología (ECyT). Universidad Nacional de San Martin (UNSAM), Consejo Nacional de Investigaciones Científicas y Tecnológicas (CONICET). Buenos Aires, Argentina. abrahanveronika@conicet.gov.ar (D) orcid.org/0000-0001-5003-4274. Google

** Licenciada en Musicoterapia. Universidad de Buenos Aires. Buenos Aires, Argentina. flaviagatto@live.com

(D) orcid.org/0000-0003-4036-5613. Google

*** Licenciada en Psicología. Doctora en Psicología. Laboratorio Interdisciplinario de Neurociencia Cognitiva (LINC). Centro de Estudios Multidisciplinarios en Sistemas Complejos y Ciencias del Cerebro (CEMSC3), Escuela de Ciencia y Tecnología (ECyT). Universidad Nacional de San Martin (UNSAM), Consejo Nacional de Investigaciones Científicas y Tecnológicas (CONICET). nadiajustel@conicet.gov. ar (iD) orcid.org/0000-0002-0145-3357. Google
} 


\title{
Effects of exposure to activating and relaxing music on the emotional state of a population of adolescents
}

\begin{abstract}
Anxiety is defined as the anticipation of a future threat with a prevalence that reaches $8 \%$ in young people around the world. Objective. The purpose of this work was to identify the effects of exposure to music on anxiety in a sample of adolescents between 10 and 14 years of age of both sexes. Materials and methods. Each of the participants completed a standardized test to assess their anxiety. Prior to the treatments, the participants completed the STAI status and trait scales, then listened to sound stimuli (relaxing, activating music, or white noise) and finally completed the anxiety status scale. Results. The results indicated that the musical stimulus with activating characteristics generated an anxiogenic effect in the participants. Conclusion. These data are relevant to the field of music therapy, a discipline that uses music with therapeutic purposes and that addresses anxiety disorders in different populations.
\end{abstract}

Keџ words

Anxiety; music; relaxing; activating; adolescents (source: MeSH, NLM).

\section{Gfeitos da exposição a música ativante e relaxante sobre o estado emocional duma população de adolescentes}

\begin{abstract}
Resumo
A ansiedade é definida como a antecipação a uma futura ameaça, com uma prevalência que alcança o $8 \%$ nas personas jovens a nível mundial. Objetivo. O propósito deste trabalho foi identificar os efeitos da exposição a música sobre a ansiedade em uma amostra de indivíduos adolescentes entre 10 e 14 anos de ambos sexos. Materiais e métodos. Cada um dos participantes completou um teste padronizado para avaliar sua ansiedade. De modo prévio aos tratamentos, os participantes completaram as escadas de estado e rasgo do STAI, depois escutaram estímulos sonoros (música relaxante, ativante ou barulho branco) e finalmente completaram a escada de estado de ansiedade. Resultados. Os resultados indicaram que o estímulo musical com características ativantes gerou um efeito antigênico nos participantes. Conclusões. Estes dados são relevantes para o campo da musicoterapia, disciplina que utiliza a música com fins terapêuticos e que aborda os transtornos de ansiedade em diferentes populações.
\end{abstract}

\footnotetext{
Palavras chave

Ansiedade; música; ativante; relaxante; adolescentes (fonte: DeCS, BIREME).
} 


\section{Introducción}

La ansiedad se define como un estado de ánimo negativo, expresado como un sentimiento de temor y tensión emocional (1). Este estado se acompañada de diversos síntomas y signos corporales, los cuales pueden desencadenarse por diversas situaciones de amenaza o peligro, real o no, ante acontecimientos o situaciones de incertidumbre, como así también frente a la posibilidad de pérdida o fracaso (o la sensación de ellas) y activa un conjunto de reacciones que implican respuestas conductuales y fisiológicas (neuronales, metabólicas y neuroendocrinas) que permiten al organismo responder al peligro de la manera más adaptativa posible (2). Su estudio y análisis implica dos conceptos fundamentales: ansiedad rasgo y el estado de ansiedad. La primera se explica como una característica de la persona y constituye una cualidad propia que diferencia la personalidad de un individuo con respecto a la de otros seres humanos; representa un estado emocional crónico que incita la realización de hábitos poco saludables. Mientras que la segunda, el estado de ansiedad, describe cómo el sujeto se siente en un momento determinado, y los síntomas están vinculados a una situación específica (1).

Según datos aportados por la Organización Mundial de la Salud (OMS) (3), la prevalencia de los Trastornos de Ansiedad (TA), es decir, los efectos prolongados de la ansiedad, alcanza el $8 \%$ en las personas jóvenes. En este sentido, cerca de 100.000.000 de personas por año padecen ansiedad en algún momento de sus vidas, pero sólo un pequeño porcentaje es diagnosticado, ya sea por falta de capacitación sanitaria o por desconocimiento de los síntomas (4).

La ansiedad constituye una emoción normal en el desarrollo entre los adolescentes y jóvenes, sin embargo, si ésta persiste, puede interferir con la vida cotidiana originando un estrés significativo y generando algún tipo de TA. La adolescencia es un periodo de vulnerabilidad debido a la presencia de todo tipo de estresores del ambiente y cambios psicológicos que provocan inestabilidad emocional (5). En este sentido, existen ciertas características y problemáticas evolutivas propias de dichas etapas del ciclo vital que podrían actuar como factores de vulnerabilidad o de riesgo de padecer ansiedad, como lo es por ejemplo embarazos no deseados, consumo de alcohol y otras sustancias psicoactivas, presión grupal y académica, conflictos intrafamiliares, carencias afectivas y económicas, y ausencia de patrones de autoridad en la familia, entre otros factores asociados al ámbito de relaciones sociales y académicas $(8,9)$.

Frente a esta problemática surge la necesidad de tratar las consecuencias negativas de la permanencia de estados ansiosos en adolescentes. Distintas técnicas psicoterapéuticas, farmacológicas o tratamientos cognitivos ofrecen alternativas de tratamiento para contrarrestar los efectos nocivos que deja en la salud la existencia de un TA (8). Dentro del abanico de posibilidades, las intervenciones que se basan en propuestas musicales han sido empleadas como una alternativa al tratamiento farmacológico para reducir la ansiedad y se ha utilizado en distintos campos como la psicología, la musicoterapia, la medicina y la odontología $(9,10,11,12)$.

La música tiene la capacidad de evocar respuestas emocionales intensas e inducir estados afectivos en las personas (10). Esta respuesta se debe a que la música actúa directamente sobre el sistema nervioso simpático disminuyendo su actividad; como resultado de lo cual el sujeto no sólo experimenta beneficios psicológicos sino también fisiológicos, como la disminución de la presión arterial, disminución de la frecuencia cardíaca y respiratoria, entre otros (13).

Dentro de la temática, se identificaron ciertas características y combinación de parámetros musicales asociados a las respuestas afectivas y emocionales (13-16). En este sentido, las piezas musicales con la capacidad de disminuir 
la activación psicofisiológica se denominan relajantes o sedantes, y se caracterizan por tener modalidades armónicas menores, ritmos y tempos lentos, timbres cálidos y cadencias resueltas (13, 17, 18). Estas, se encuentran asociadas a una disminución del pulso cardíaco, el ritmo en la respiración y la presión sanguínea $(19,20)$. Por su parte, la música activante, es decir, piezas con modalidades armónicas mayores, ritmos rápidos, timbres estridentes, $\mathrm{y} / \mathrm{o}$ con presencia de estructuras musicales que rompen con la expectativa del oyente producen un incremento de los aspectos fisiológicos presentados $(21,22)$. En uno de los estudios citados por Chanda y Levitin (20), los investigadores seleccionaron música académica considerada como relajante (60 pulsos/ minuto) y música electrónica considerada como activante (130 pulsos/minuto). En este estudio, los participantes (quienes expresaron no tener preferencia musical alguna), con la exposición a música electrónica tuvieron un incremento de cortisol, acetilcolina, prolactina, hormonas de crecimiento y niveles de norepinefrina consistentemente con aumento de los niveles del eje HPA (hipotalámico-hipofisario-adrenal) y la actividad del sistema nervioso parasimpático (20, 23).

Una disciplina que utiliza la música con fines terapéuticos es la musicoterapia, la cual, además, presenta investigaciones empíricas con resultados favorables sobre la implementación de intervenciones basadas en música para contrarrestar la ansiedad en distintas poblaciones $(8,13,24-31)$. Sin embargo, varios de los antecedentes están focalizados en pacientes internados o expuestos a una intervención médica $(26,27)$ y utilizando la intervención musical durante los procedimientos (28). Aquí nos proponemos abordar la temática con población adolescente en contexto educativo y sin diagnóstico de ansiedad patológica.

Teniendo en cuenta los antecedentes presentados, el objetivo de este trabajo fue evaluar el efecto de la escucha de una pieza musical relajante y otra activante sobre el nivel de ansiedad en adolescentes. Respecto de los antecedentes sobre los efectos fisiológicos, dependiendo de las características musicales de los estímulos $(20,32)$, se esperaba que la exposición a música relajante disminuyera la ansiedad estado en los adolescentes, mientras que la música con parámetros activantes la elevase.

\section{Método}

\section{Diseño y población de estudio}

Se realizó un estudio cuasi-experimental que buscó establecer el efecto de la escucha musical con características relajantes y activantes sobre la ansiedad estado y rasgo en adolescentes. Participaron de este trabajo 32 adolescentes (15 varones y 17 mujeres) de entre 10 y 14 años $(M=11.96 ; D E=.19)$, el $90 \%$ eran diestros, pertenecientes a un Grupo Scout y Colegio Santa Teresa del Niño Jesús de San Isidro, provincia de Buenos Aires. Los sujetos fueron asignados de modo aleatorio, bajo distribución verbal al azar a cada uno de los grupos. El trabajo se rigió de acuerdo a las normas éticas vigentes (33). Se les solicitó a los padres o tutores de los participantes que firmasen un consentimiento informado en donde se detallaban los propósitos del estudio y el compromiso de asegurar el anonimato y la confidencialidad de los datos. Además, se les informó a los adolescentes que, si lo deseaban, podían abandonar el estudio en cualquier momento de su realización.

\section{Instrumentos de evaluación}

Cuestionario Sociodemográfico. Cuestionario que indaga acerca de la edad, sexo, lateralidad, consumo de sustancias psicoactivas o medicamentos, enfermedades relevantes y años de educación académica. Se incorporaron dos preguntas en cuanto a lo musical: si disfrutaba de la música y cuáles eran los estilos musicales predilectos. 
Inventario de Ansiedad Estado Rasgo. Uno de los autoinformes más utilizados y evaluados en la literatura para la evaluación de la ansiedad estado y rasgo en población general y clínica es el Inventario de ansiedad estado-rasgo (STAI por su sigla en inglés: State - Trait Anxiety Inventory) (34). El STAI es un cuestionario cuyo objetivo es evaluar el nivel de ansiedad y la predisposición del sujeto a responder al estrés y se compone de 40 ítems que se encuentran divididos en dos sub grupos. Los primeros 20 ítems pertenecen a la subescala "estado", formados por frases que describen cómo se siente la persona en el momento en que realiza el test, y la otra mitad pertenece a la subescala "rasgo" en donde el sujeto selecciona las respuestas en pos de cómo se siente habitualmente (1). Cada subescala se conforma por los ítems en un sistema de respuesta Likert de 4 puntos según la intensidad $(0=$ casi nunca/nada; $1=$ algo/a veces; $2=$ bastante/a menudo; $3=$ mucho/casi siempre).

\section{Tratamientos}

Música relajante. Los sujetos escucharon el Canon en Re mayor de Johann Pachelbel.

Música activante. Los sujetos escucharon el Cuarteto de cuerdas $\mathrm{N}^{\circ} .10$ en Si mayor. Op. 2, No. 6, 3er movimiento de Joseph Haydn.

Ruido blanco. Los sujetos escucharon ruido blanco con una intensidad media (señal aleatoria).

La elección de las piezas de tipo relajante y activante se basó en la metodología del estudio conducido por los autores Kreutz, Ott, Teichmann, Osawa, y Vaitl (35), mientras que el ruido blanco fue elegido tomando en consideración la investigación de Rickard, Wing Wong y Velik (36). Las piezas fueron reproducidas bajo un archivo de audio formato de intercambio WMA, con un parlante inalámbrico Flip 3 JBL. La intensidad de reproducción fue establecida por los investigadores, de acuerdo a las características del ambiente, con un volumen de intensidad medio/alto.

\section{Procedimiento}

El estudio tuvo lugar en el salón de usos múltiples de la institución, espacio caracterizado por la ausencia de ruido ambiente excesivo. En primer lugar, se entregó el consentimiento informado a los padres; una vez chequeada la autorización y el asentimiento de los adolescentes, los participantes completaron el cuestionario sociodemográfico. A continuación, se explicó el procedimiento y los participantes completaron en primera instancia la subescala "ansiedad estado" del STAI y luego la sub-escala "ansiedad rasgo" (medida pre tratamiento). Luego, se dividió a los participantes a través asignación verbal al azar y se los invitó a adoptar una posición cómoda, a no hablar con sus compañeros y a concentrarse en lo que iban a escuchar. Un grupo de participantes fue expuesto al estímulo musical activante, un grupo diferente de sujetos al estímulo musical relajante y, por último, otro grupo de adolescentes escuchó el estímulo control (ruido blanco). La exposición tanto a música (ambos estímulos: relajante y activante) como al ruido blanco tuvo una duración de 4 minutos. Inmediatamente finalizada la escucha, los participantes completaron la sub-escala de ansiedad estado (medida post tratamiento).

\section{Análisis de datos}

Para llevar a cabo los análisis descriptivos e inferenciales se utilizó el software SPSS Statistics 17.0.2. En primera instancia se analizaron los supuestos de normalidad y homogeneidad de la muestra, para lo cual se utilizaron los estadísticos de Levenne y Shapiro Wilk. Debido a que la muestra cumplía ambos supuestos, las medidas de ansiedad estado y rasgo se analizaron independientemente con un análisis de varianza (ANOVA) de medidas repetidas (MR). El factor intersujeto fue Tratamiento (Activante, Relajante y Control), mientras que Tiempo (Pre y Post) para la ansiedad estado fue la MR.

Por otra parte, se realizó un análisis dependiendo del sexo de los participantes, con el fin de identificar 
posibles diferencias en los valores de ansiedad atribuibles a la variable cualitativa. Las medidas de ansiedad rasgo y estado de ansiedad (pre y post) se analizaron independientemente con un análisis de varianza (ANOVA) de medidas repetidas (MR). El factor intersujeto fue Sexo (Mujeres vs Hombres), mientras que Tiempo (Pre y Post) para la ansiedad estado fue la MR.

Para analizar los efectos principales y las interacciones significativas, se realizaron pruebas Post-hoc, estimándose aceptable el nivel de significación de .05. Se utilizó el Eta cuadrado parcial $\left(\eta^{2} \mathrm{p}\right)$ para estimar el tamaño del efecto.

\section{Conflicto de intereses}

El manuscrito fue preparado y revisado por las autoras, quienes declaran que no existe ningún conflicto de intereses que ponga en riesgo la validez de los resultados presentados.

\section{Resultados}

\section{Características de la muestra}

La muestra final estuvo conformada por 32 adolescentes (15 varones y 17 mujeres) con una edad de entre 10 y 14 años $(M=11.9 ; D S=0.19)$. A través del cuestionario sociodemográfico se indagó sobre las preferencias musicales de los participantes, siendo la cumbia (23\%) y el pop (22\%) los estilos musicales mayormente reportados. En la Figura 1 se puede observar la distribución de los estilos musicales reportados. A través de estos datos se corrobora que la predilección musical no es una variable interviniente en los resultados de la modulación emocional, ya que el género clásico (característico de las piezas musicales utilizadas en el presente estudio) no aparece entre los reportados.

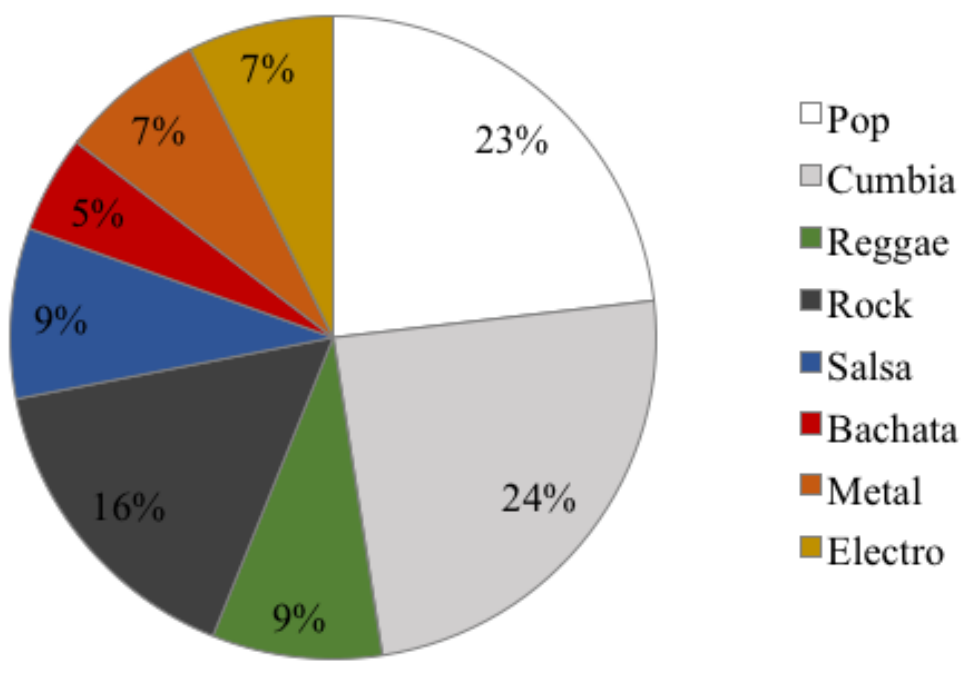

Figura 1. Estilos Musicales predilectos reportado por los participantes.

Fuente: las autoras. 
Teniendo en cuenta los antecedentes que muestran que las mujeres son más vulnerables a desarrollar trastornos de ansiedad que los hombres, aproximadamente entre 2 a 3 veces más (28), se realizó un primer análisis en cuanto a las posibles diferencias en los valores de ansiedad dependiendo del sexo de los participantes (mujeres vs hombres).
Los análisis estadísticos no reportaron diferencias significativas en las medidas de ansiedad rasgo y estado (tanto en las mediciones pre como post tratamiento; $p>.05$ ). De esta forma se corrobora que el sexo de los participantes no es una variable interviniente en la modulación de la ansiedad producto de la percepción musical (Figura 2).

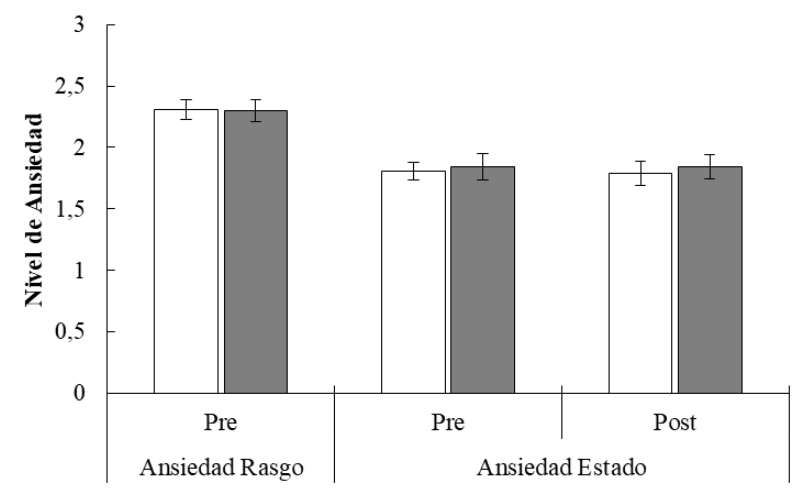

Figura 2. Nivel de ansiedad de acuerdo al sexo de los participantes.

Fuente: las autoras.

\section{Ansiedad Rasgo}

La ansiedad rasgo es una característica de la persona y constituye una cualidad propia que diferencia la personalidad de un individuo con respecto a la de otros seres humanos; representa un estado emocional crónico que incita la realización de hábitos poco saludables que perpetúan la exacerbación de los valores normales (1). En relación a esta variable, el análisis estadístico no mostró ningún efecto principal o interacción estadísticamente significativa $p>.05$ (ver Figura $3)$, lo que indica que los tres grupos presentaron el mismo nivel de ansiedad.

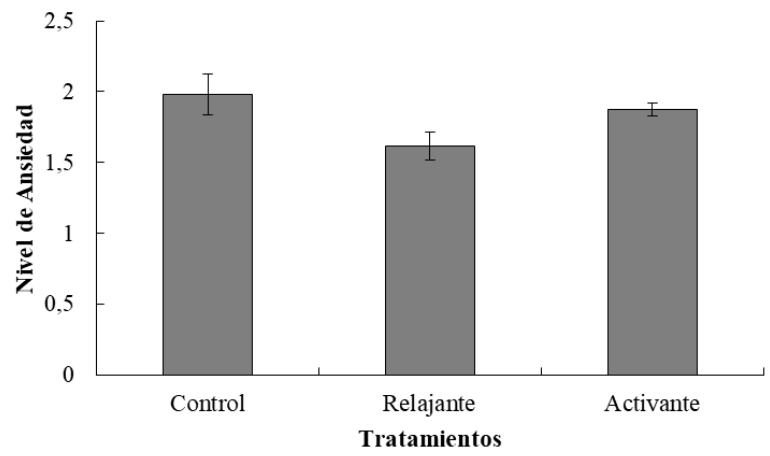

Figura 3. Ansiedad Rasgo. Pre: Resultados del autoinforme de modo previo a la exposición al estímulo musical. Post: Resultados del autoinforme de modo posterior a la exposición al estímulo musical. Las líneas verticales representan el error estándar. 


\section{Ansiedad Estado}

Como se refirió anteriormente, la ansiedad estado describe cómo el sujeto se siente en un momento determinado (1). El análisis estadístico, indicó un efecto principal de Tratamiento $F(1,29)=4.94, p$ $=.014, \eta^{2} \mathrm{p}=.25$, y una significación de la doble interacción Tiempo x Tratamiento $F(2,29)=4.14$, $p=.026, \eta^{2} \mathrm{p}=.22$. Los correspondientes Post-hoc mostraron que, en las medidas obtenidas antes de la exposición a los tratamientos, el grupo control tuvo valores más altos de ansiedad que el grupo relajante. Por otra parte, luego de la exposición al tratamiento se encontró que el grupo de sujetos que escuchó música activante presentó mayor nivel de ansiedad (ver Figura 4).

Por otra parte, se realizó un análisis adicional comparando el nivel de ansiedad rasgo (pre tratamiento) vs el estado de ansiedad (post tratamiento) de los participantes, con el objetivo de identificar posibles diferencias entre ambas medidas. El análisis estadístico indicó un efecto principal de Tiempo $F(1,29)=50.520, p<$ $.001, \eta^{2} \mathrm{p}=.63$, y una significación de la doble interacción Tiempo x Tratamiento $F(2,29)=3.602$, $p=.040, \eta^{2} \mathrm{p}=.19$. Los correspondientes Post-hoc mostraron que los niveles de ansiedad son menores luego de la exposición a los tratamientos. Por otra parte, la doble interacción indicó que, en las medidas del estado de ansiedad respecto del rasgo, los participantes que escucharon la pieza activante presentaron mayor nivel de ansiedad que los del grupo control y relajante, mientras que el control presentó mayores niveles de ansiedad que los que escucharon la pieza relajante (ver Figura 5).

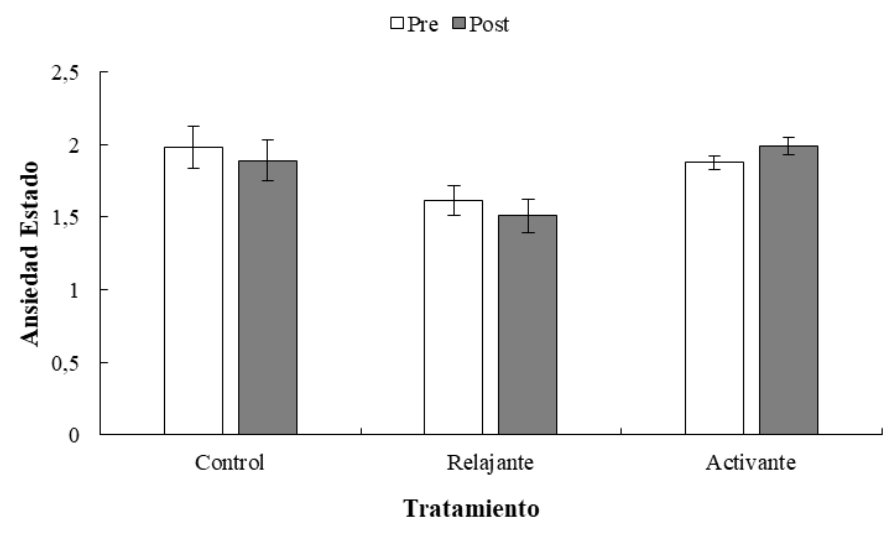

Figura 4. Ansiedad Estado. Pre: Resultados del autoinforme de modo previo a la exposición al estímulo musical. Post: Resultados del autoinforme de modo posterior a la exposición al estímulo musical. Las líneas verticales representan el error estándar.

Fuente: las autoras. 


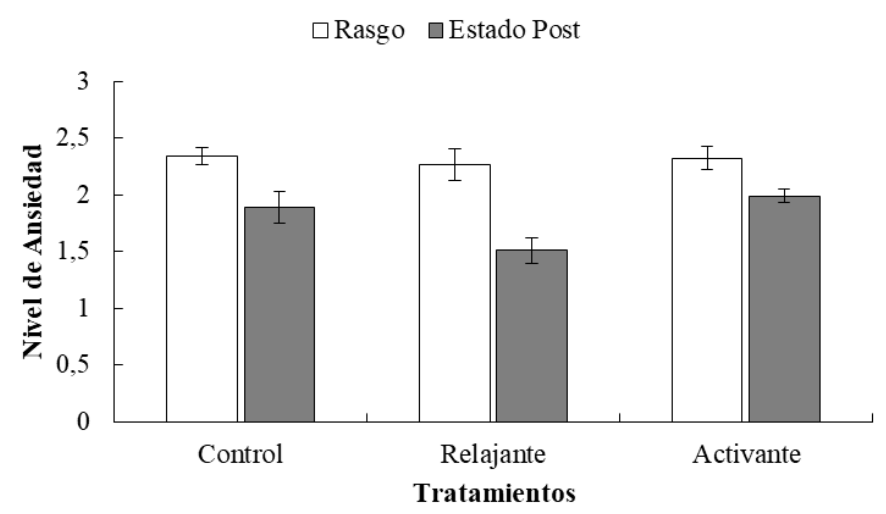

Figura 5. Análisis adicional. Ansiedad rasgo vs Estado de ansiedad (post tratamiento). Las líneas verticales representan el error estándar.

Fuente: las autoras.

\section{Discusión}

El objetivo de esta investigación fue dilucidar el efecto de la exposición a música en adolescentes con el fin de modular el nivel de ansiedad que presentaban al momento del estudio. Teniendo en cuenta nuestra hipótesis inicial, se esperaba que la exposición a música relajante disminuyera el estado de ansiedad en los adolescentes, mientras que la música con parámetros activantes la elevase. Estas hipótesis fueron corroboradas sólo parcialmente.

Los resultados obtenidos en este estudio señalan que la música con características activantes posee un efecto ansiogénico. En este sentido, y apoyándonos en la revisión de la literatura que realizaron Chanda y Levitin (20), podrían destacarse las condiciones paramétricas bajo las cuales el estímulo activante induciría mayores niveles de ansiedad. La exposición a música con características activantes ha mostrado producir un incremento de sustancias químicas como el cortisol, acetilcolina, prolactina, hormonas de crecimiento, y niveles de norepinefrina consistentemente con aumento de los niveles del eje HPA y la actividad del sistema nervioso simpático $(20,24)$, algunos de estos factores son considerados elementos constitutivos de los denominados trastornos de ansiedad (38). Bajo estos resultados e inferencias, el uso de música activante no sería una opción adecuada para el abordaje de población con TA, empero puede ser pensada e implementada en otro tipo de problemáticas relacionadas a los estados emocionales, como por ejemplo la depresión, donde resulta necesario aumentar los niveles de activación y excitación fisiológica del paciente. Sin embargo, para arribar a este tipo de inferencias, es necesario contar con futuros estudios con mediciones periféricas indicadores de ansiedad, como lo son por ejemplo la frecuencia respiratoria, cardíaca, o la respuesta galvánica $(24,39)$, con el fin de establecer la relación entre los estímulos musicales y los parámetros fisiológicos.

La ausencia de efecto por parte de la pieza musical con características relajantes es un resultado inesperado, teniendo en cuenta los antecedentes dentro de la literatura, los cuales asocian estos estímulos con una disminución del pulso cardíaco, el ritmo en la respiración y la presión sanguínea, y la consecuente disminución de la ansiedad (1720). Existen diferentes ideas para justificar este 
resultado. En principio, un aspecto no menor que caracteriza nuestra muestra es la ausencia de niveles de ansiedad patológicos o anormales. En este sentido, puede que la música no presente el efecto esperado al no contar con un estado psicofisiológico de ansiedad el cual requiera ser modulado.

Por otra parte, respecto de la implementación de las intervenciones y tratamiento, una crítica constante a los estudios sobre el efecto de la música, tanto a nivel fisiológico, neuroanatómico como cognitivo, es la falta de control metodológico en los diseños. Los antecedentes sobre la temática sugieren que las intervenciones musicales son uno de los métodos que alivian la ansiedad, empero no se describen las características concretas del tipo de intervención o estímulos sonorosos utilizados (40). Bajo estas incongruencias puede que las obras musicales utilizadas en nuestro estudio, al compararlas con las investigaciones previas, no sean las mismas, lo cual podría dejar entrever una posible justificación ante la discrepancia. En este sentido, reforzamos lo expuesto por Robb, Burns y Carpenter (41), quienes plantean la importancia y la necesidad de contar con la descripción transparente y específica de los informes y procedimientos sobre intervenciones basadas en música, con el fin último de poder replicar los estudios y aplicarlos correctamente.

Continuando con el foco en el tipo de herramienta utilizada, nuestro estudio se caracterizó por la exposición de un estilo musical no predilecto por la muestra de adolescentes. Este aspecto fue considerado positivamente en nuestro diseño ya que permitía aislar el efecto del estímulo musical evitando la incorporación de variables intervienes, como lo es la preferencia musical. Sobre este tópico, un estudio llevado a cabo por Wu, Huang, Lee, Wang y Shih (40) utilizó piezas musicales seleccionadas por los participantes, de acuerdo a las preferencias personales, encontrando diferencias en los indicadores fisiológicos de ansiedad. En este punto, sería interesante replicar nuestro estudio, bajo la misma metodología de investigación implementada, pero utilizando estímulos seleccionados por los adolescentes, de acuerdo a la edad y el contexto sociocultural.

Finalmente, se puede pensar que para lograr un efecto relajante a través de la música son necesarios tiempos de exposición más prolongados para lograr un impacto fisiológico. En nuestro estudio la percepción musical fue de 4 minutos, futuros estudios podrían orientarse hacia el uso de diferentes ventanas temporales.

Es interesante señalar que el presente estudio es de una aplicación aguda y puntual, sin seguimiento de caso, por lo que se necesitan más estudios para poder dilucidar el rol de estímulos relajantes de modo crónico y con seguimiento de la ansiedad estado a lo largo de diferentes períodos de tiempo. Aunque se ha establecido la eficacia de los efectos ansiolíticos de la música para la ansiedad preoperatoria, los hallazgos sobre el uso de escuchar música para la ansiedad no son concluyentes. Es posible que sean necesarias intervenciones ofrecidas por un musicoterapeuta capacitado para optimizar el impacto de la música. Las intervenciones de musicoterapia se individualizan de acuerdo a las necesidades específicas de cada paciente o grupo de pacientes $\mathrm{y}$, además, los musicoterapeutas pueden enseñar a los usuarios y pacientes habilidades de manejo de la ansiedad utilizando la música como herramienta de afrontamiento (42).

La comprensión de las bases neurobiológicas, neurofisiológicas y neuropsicológicas de la experiencia musical es importante no sólo desde el punto de vista de la promoción de la investigación y la adquisición de conocimientos básicos, sino también en vista de la aplicación potencial de la música en la práctica clínica (43). Sin embargo, y en relación a los resultados obtenidos en este trabajo, resulta de suma importancia conocer los efectos específicos de cada estimulo musical con el cual se trabaja, ya que se ha podido observar que la música, dependiendo de las características de sus 
parámetros sonoros, presentanefectos diferenciales, en este caso, sobre la ansiedad. Resulta pertinente continuar indagando sobre los efectos neuronales, fisiológicos y comportamentales de cada uno de los parámetros del discurso musical (como timbre o melodía), pero también es relevante el estudio de la combinación de ellos, así como el efecto de diversas piezas musicales.

En otro orden de ideas, nuestro estudio presenta una limitación importante, determinada por las diferencias intergrupales iniciales en los niveles de ansiedad estado, ya que se encontraron diferencias entre el grupo expuesto a ruido blanco (control) y aquellos que escucharon música relajante. En este sentido contar con una muestra inicial homogénea es de suma importancia para las comparaciones posteriores, aspecto que será tenido en cuenta en futuras investigaciones.

\section{Conclusiones}

El presente estudio indagó sobre el efecto de la exposición a música en adolescentes con el fin de modular la ansiedad, encontrando que la música con características activantes posee un efecto ansiogénico.

No deja de ser una realidad que día a día son cada vez más las preocupaciones y miedos por los que un adolescente transita (6). Actualmente, la ansiedad se traduce no sólo en cumplir con los parámetros educativos normados sino con la necesidad de formar parte de un grupo de pertenencia, los miedos acerca de las situaciones familiares, algunos tipos de violencia encontrados en las distintas instituciones por las que atraviesa y sumado a eso la tendencia a sentir la obligación de saber qué hacer con sus vidas. Se entiende por lo tanto que hay que tener en cuenta el contexto social del sujeto a ser tratado. La música es una herramienta más de contención que puede ser usada en la red que necesita el adolescente, aspecto que orientó el presente trabajo.

Las posibilidades en torno a las intervenciones musicales brindan no sólo opciones ecológicas, simples y accesibles a la comunidad (44), sino que también proveen propuestas motivadoras para la población adolescente. La evidencia preliminar parece indicar un posible papel de la musicoterapia en el manejo clínico de una variedad de trastornos neurológicos y condiciones psiquiátricas (45).

\section{Agradecimientos}

Agradecemos al Grupo Scout y a alumnos de 6to grado del Colegio Santa Teresa del Niño Jesús de San Isidro, provincia de Buenos Aires, por participar del presente estudio. 


\section{Referencios bibliográficos}

1. Spielberger C. STAI: Manual for the State-Trait Anxiety Inventory (Self Evaluation Questionnaire). Madrid: Tea Ediciones; 2011

2. Duval F, Gonzalez F, Rabia H. Neurobiología del estrés. Rev. Chil. de Neuro-Psiquiat. 2010; 48 (4): 307-318. DOI: http://dx.doi.org/10.4067/S0717-92272010000500006

3. Organización Mundial de la Salud (OMS) [Sitio web mundial]. La inversión en el tratamiento de la depresión y la ansiedad tiene un rendimiento del 400\% [Comunicado de prensa en internet]. Disponible en: https://www.who.int/mediacentre/news/releases/2016/depression-anxietytreatement/es/

4. Acosta AF, Rodríguez AL, Lotero JA. Epidemiología de los trastornos de ansiedad y depresión en adolescentes de una población rural. Rev. posgrado VIa. Cátedra Med. 2008; 184: 4-9. Disponible en https://med.unne.edu.ar/revistas/revista184/2_184.pdf

5. Sánchez-Gómez M, Oliver A, Adelantado-Renau M, Breso E. Inteligencia emocional y ansiedad en adolescentes: una propuesta práctica en el aula. Quaderns digitals: Revista de Nuevas Tecnologías y Sociedad. 2020; 91: 74-89. Disponible en http://www.quadernsdigitals.net/index. php?accionMenu=hemeroteca.VisualizaArticuloIU.visualiza\&articulo_id=11544

6. Czernik GE, Giménez NL, Almirón LM, Larroza GO. Ansiedad Rasgo-Estado en una Escuela de Formación Profesional de la ciudad de Resistencia (Chaco). Comunicaciones Científicas y Tecnológicas. Universidad Nacional del Nordeste: Corrientes (Argentina); 2005. Disponible en https://scholar.google.com/citations?user=7Cmxz0UAAAAJ\&hl=es

7. Herrera-Gutiérrez E, Brocal-Pérez D, Sánchez-Marmol DJ, Rodríguez-Dorantes JM. Relación entre actividad física, depresión y ansiedad en adolescentes. Cuad. de Psicol. del Deporte. 2013: 12 (2), 31-38. Disponible en https://revistas.um.es/cpd/article/view/177731

8. Capafons A. Tratamientos psicológicos eficaces para la ansiedad generalizada. Psicothema. 2001; 13 (3): 442-446. Disponible en http://www.psicothema.com/psicothema.asp?id=466

9. Ilkkaya NK, Ustun FE, Sener EB, Kaya C, Ustun YB, Koksal E, Ozkan F. The effects of music, white noise, and ambient noise on sedation and anxiety in patients under spinal anesthesia during surgery. J Perianesth Nurs. 2014; 29 (5): 418-426. DOI: https://doi.org/10.1016/j.jopan.2014.05.008

10. Juslin PN. Musical Emotions Explained. Oxford: Oxford University Press; 2019.

11. Kamioka H, Tsutani K, Yamada M, Park H, Okuizumi H, Tsuruoka K, et al. Effectiveness of music therapy: a summary of systematic reviews based on randomized controlled trials of music interventions. Patient Prefer Adherence. 2014; 8: 727-754. DOI : https://doi.org/10.2147/PPA. S61340

12. Norouzi F, Keshavarz M, Seyed-Fatemi N, Montazeri A. The impact of kangaroo care and music on maternal state anxiety. Complement Ther Med. 2013; 21 (5): 468-472. DOI: https://doi.org/10.1016/j. ctim.2013.07.006

13. Rickard N. Intense emotional responses to music: A test of the physiological arousal hypothesis. Psychology of Music. 2004; 32 (4): 371-388. DOI: https://doi.org/10.1177/0305735604046096

14. Grocke D, Wigram T. Receptive Methods in Music Therapy Techniques and Clinical Applications for Music Therapy Clinicians, Educators and Students. Philadelphia: Jessica Kingsley Publishers; 2007 
15. Gómez-Scarpetta R, Arismendy L, Cabra-Sosa L, Vargas C, Becerra N. Musicoterapia para el control de ansiedad odontológica en niños con síndrome de Down. Hacia. Promoc. Salud. 2012; 17 (2): 13-24. Disponible en https://www.redalyc.org/articulo.oa?id=309126826002

16. Zain J. Escuchar el silencio. Musicoterapia Vibroacústica. Buenos Aires: Kier; 2014.

17. Aldridge D. Music therapy research: A review of references in the medical literature. The Arts in Psychotherapy. 1993; 20 (1): 11-35. Disponible en http://www.wfmt.info/Musictherapyworld/ modules/archive/stuff/papers/mtreview.pdf

18. Justel N, O'Conor J, Rubinstein W. Emotional memory modulation through music in older people: A preliminary study. Interdisciplinaria. 2015, 32 (2), 247-259. Disponible en https://www.redalyc. org/jatsRepo/180/18043528003/html/index.html

19. Bernardi L. Dynamic interaction between musical, cardiovascular, and cerebral rhytms in humans. Circulation. 2009; 119 (25): 3171-3180. DOI: https://doi.org/10.1161/circulationaha.108.806174

20. Chanda ML, Levitin D. The neurochemistry of music. Trends Cogn Sci. 2013; 17 (4): 179-193. DOI: https://doi.org/10.1016/j.tics.2013.02.007

21. Justel N, Rubinstein W. La exposición a la música favorece la consolidación de los recuerdos. Boletín de Psicología. 2013, 109, 73-83. Disponible en https://dialnet.unirioja.es/servlet/ articulo? codigo $=4495380$

22. Justel N, Diaz-Abrahan V, Castro C, Rubinstein W. Efecto de la música sobre la memoria emocional verbal. Anuario de Psicología. 2016, 22 (2), 297-301. URL: https://www.redalyc.org/articulo. oa? id $=369147944072$

23. Gerra G, Zaimovic A, Franchini D, Palladino M, Giucastro G, Reali N, et al. Neuroendocrine responses of healthy volunteers to 'techno-music': Relationships with personality traits and emotional state. Int J Psychophysiol. 1998; 28 (1): 99-111. DOI: https://doi.org/10.1016/s01678760(97)00071-8

24. Blood AJ, Zatorre RJ, Evans PB. Emotional responses to pleasant and unpleasant music correlate with activity in paralimbic brain region. Nat Neurosci. 1999; 2 (4): 382-387. DOI: https://doi. org/10.1038/7299

25. Chan Y, Lee $P$. The use of music to reduce anxiety for patients undergoing colposcopy: a randomized trial. Gynecol Oncol. 2003; 91 (1): 213-227. DOI: https://doi.org/10.1016/s0090-8258(03)00412-8

26. Walker JC, McNaughton A. Does listening to music preoperatively reduce anxiety? An evidencebased practice process for novice researchers. British journal of nursing. 2018, 27 (21), 1250-1254. DOI: https://doi.org/10.12968/bjon.2018.27.21.1250

27. Wu PY, Huang ML, Lee WP, Wang C, Shih WM. Effects of music listening on anxiety and physiological responses in patients undergoing awake craniotomy. Complementary therapies in medicine. 2017, 32, 56-60. DOI: https://doi.org/10.1016/j.ctim.2017.03.007

28. Ince $\mathrm{S}$, Çevik $\mathrm{K}$. The effect of music listening on the anxiety of nursing students during their first blood draw experience. Nurse education today. 2017, 52, 10-14. DOI: https://doi.org/10.1016/j. nedt.2017.02.009

29. Lahmann C, Schoen R, Henningsen P, Ronel J, Muehlbacher M, Loew T, et al. Brief relaxation versus music distraction in the treatment of dental anxiety: A randomized controlled clinical trial. $\mathrm{J}$ Am Dent Assoc. 2008; 139: 317-324. DOI: https://doi.org/10.14219/jada.archive.2008.0161

30. McKinney C, Antoni M, Kumar M, Tims F, McCabe P. Effects of guided imagery and music (GIM) therapy on mood and cortisol in healthy adults. Health Psychol. 1997; 16 (4): 390-400. DOI: https:// doi.org/10.1037//0278-6133.16.4.390 
31. Nieto-Romero RM. Efectos de la musicoterapia sobre el nivel de ansiedad del adulto cardiópata sometido a resonancia magnética. Enfermería Universitaria. 2017; 14 (2): 88-89. DOI: https://doi. org/10.1016/j.reu.2017.02.002

32. Zatorre RJ, Chen JL, Penhune VB. When the brain plays music: auditory-motor interactions in music perception and production. Nat Rev Neurosci. 2007; 8 (7): 547-558. DOI: https://doi. org/10.1038/nrn2152

33. Asociación Médica Mundial (WMA). Declaración de Helsinki de la AMM - Principios éticos para las investigaciones médicas en seres humanos [Internet]. Consultado 3 de oct. 2018. Disponible en: https://www.wma.net/es/policies-post/declaracion-de-helsinki-de-la-amm-principios-eticos-paralas-investigaciones-medicas-en-seres-humanos/

34. Fonseca-PedreroE, Paino M, Sierra-Baigrie S, Lemos-GiráldezS, MuñizJ.Propiedades psicométricas del Cuestionario de ansiedad estado-rasgo (STAI) en universitarios. Psicología Conductual. 2012; 20 (3): 547-561. Disponible en: https://dialnet.unirioja.es/servlet/articulo?codigo=4131885

35. Kreutz G, Ott U, Teichmann D, Osawa P, Vaitl D. Using music to induce emotions: Influences of musical preference and absorption. Psychology of Music. 2008; 36 (1): 100-127. DOI: https://doi. org/10.1177/0305735607082623

36. Rickard N, Wing-Wong W, Velik L. Relaxing music counters heightened consolidation of emotional memory. Neurobiol Learn Mem. 2012; 97 (2): 220-228. DOI: https://doi.org/10.1016/j. nlm.2011.12.005

37. Carrasco-Galán, I. y Espinar-Fellmann, I. (2008). Trastornos de ansiedad y género. Mente y Cerebro, 31, 12-21. Disponible en: https://www.investigacionyciencia.es/revistas/mente-y-cerebro/ psicologa-de-la-gestacin-466/trastornos-de-ansiedad-y-gnero-502

38. Sadek N, Nemeroff C. Update on the Neurobiology of Depression [Internet]. Consultado 3 de oct. 2018. Disponible en http://www.medscape.org/viewarticle/412866/

39. Roy M, Peretz I, Rainville P. Emotional valence contributes to music-induced analgesia. Pain. 2008, 134 (1-2), 140-147. DOI: https://doi.org/10.1016/j.pain.2007.04.003

40. Robb SL, Burns DS, Carpenter JS. Reporting guidelines for music-based interventions. J Health Psychol. 2011; 16 (2): 342-352. DOI: https://doi.org/10.1177/1359105310374781

41. Wu P, Huang M, Lee W, Wang C, Shih W. Effects of music listening on anxiety and physiological responses inpatients undergoing awake craniotomy. Complementary Therapies in Medicine. 2017; 32, 56-60. DOI: https://doi.org/10.1016/j.ctim.2017.03.007

42. Bradt J, Teague A. Music interventions for dental anxiety. Oral diseases. 2018; 24 (3), 300-306. Disponible en https://doi.org/10.1111/odi.12615

43. Abrahan V, Justel N. La improvisación musical. Una mirada compartida entre la musicoterapia y las neurociencias. Psicogente. 2015; 18 (34): 372-384. Disponible en: http://publicaciones. unisimonbolivar.edu.co/rdigital/ojs/index.php/psicogente/article/view/512/0

44. Bugos JA, Perlstein WM, McCrae CS, Brophy TS, Bedenbaugh PH. Individualized piano instruction enhances executive functioning and working memory in older adults. Aging Ment Health. 2007; 11 (4): 464-471. DOI: https://doi.org/10.1080/13607860601086504

45. Boso M, Politi P, Barale F, Emanuele E. Neurophysiology and neurobiology of the musical experience. Funct Neurol. 2006; 21 (4): 187-191. Disponible en: https://pubmed.ncbi.nlm.nih. gov/17367577/ 